\section{Disnea persistente sin causa orgánica: un caso de base psicosomática}

\section{Sr. Editor}

La disnea es un término clínico que describe la sensación subjetiva de respiración dificultosa y que abarca un amplio rango de síntomas, tales como el acortamiento de la respiración o el aumento de la frecuencia respiratoria. ${ }^{1}$ Con independencia de sus mayoritarias causas orgánicas, la relación causal entre disnea y síntomas psicológicos, principalmente ansiedad y depresión, ha sido bien establecida ${ }^{2}$ y vinculada principalmente a hiperventilación. ${ }^{3}$

Otra relación bien conocida es la expresión psicosomática de la disnea, ${ }^{4}$ la cual afecta principalmente a adultos jóvenes y coexiste habitualmente con hiperventilación y otros síntomas inespecíficos (cardíacos, etcétera). Estos pacientes refieren un elevado nivel de ansiedad y describen, en comparación con pacientes asmáticos y otros enfermos pulmonares, una intensidad superior de disnea (escala subjetiva de automedición) y una menor capacidad para contener la respiración luego de una inspiración profunda (breath-holding time) ${ }^{5}$

Presentamos el caso de un hombre de 65 años, deportista habitual (travesías de alta montaña), no fumador e hipertenso (enalapril $10 \mathrm{mg} / \mathrm{día}$ ). El paciente consultó por disnea de medianos esfuerzos y ocasionalmente de reposo, sin sibilancias y sin síntomas cardíacos. La disnea había empeorado en los últimos dos años, lo que el paciente vinculaba a un accidente isquémico transitorio (AIT) acaecido a 5,400 metros de altitud en un campamento base de Nepal (AAS 300 mg/día), y desde entonces, había abandonado la práctica deportiva por disnea.
Al momento de la consulta, el paciente no presentaba secuelas neurológicas y mantenía un índice de masa corporal estable en los últimos meses (IMC 30). $\mathrm{Su}$ radiografía de tórax, electrocardiograma, datos analíticos (incluyendo perfil tiroideo), oximetría en reposo y prick-test eran satisfactorios. La espirometría arrojó los siguientes datos normales: $\mathrm{FEV}_{1} / \mathrm{FVC}$ $80 \%$ (referencia 73\%), $\mathrm{FEV}_{1} 2.611$ (97\%, referencia 2.71 1) y FVC 3.231 (87\%, referencia 3.74 1). Los valores de TLC (total lung capacity), RV (residual volume) y FRC (functional residual capacity) fueron, respectivamente, $112 \%, 98 \%$ y $91 \%$ (estándares). El coeficiente de difusión de monóxido de carbono (KCO) fue del 95\% (normal).

Se realizó, además, una gasometría venosa que resultó no patológica ( $\mathrm{pH} 7.41$, $\mathrm{pCO}_{2} 45$, bicarbonato 23 ) y una prueba de la marcha de 6 minutos con una distancia recorrida de 360 metros (saturación basal 98\%, saturación mínima 95\%). Desde el punto de vista cardiológico, se efectuó una prueba de esfuerzo (protocolo de Bruce), alcanzando el paciente una carga de 12 METS, sin evidenciar alteraciones clínicas y eléctricas. Sólo se detectó (ecocardiograma) una cardiopatía hipertensiva ligera (septum $13 \mathrm{~mm}$ ), sin depresión funcional ni alteraciones de la contractilidad.

Fue remitido a psicología, orientándose como «disnea funcional». La anamnesis psicológica reveló múltiples experiencias vitales asociadas a episodios traumáticos de «ahogos» (ejemplos: enfermedad respiratoria crónica padecida por su madre, sensación claustrofóbica al quedarse atrapado en una cueva a los 16 años o el haber presenciado el ahogamiento de un amigo en un río a los 17 años). Resaltaba la necesidad de «ambientes amplios y con aire puro» (montañismo), razón que incluso lo condujo a evitar utilizar ascensores, camisas abotonadas hasta el cuello o ceñirse la corbata. Estos episodios de «ahogos» comenzaron hacia los 44 años (divorcio) y continuaron siendo relativamente infrecuentes (estrés, ansiedad), hasta que se generalizaron luego del AIT (63 años). Desde el punto de vista psicológico, este agravamiento fue atribuido a una crisis vital (edad).

Bajo el término «síndromes psicosomáticos, somatizaciones y alteraciones somatoformes», se agrupan entidades donde el proceso psicológico juega un papel sustancial en la etiología de la enfermedad de algunos pacientes. ${ }^{6}$ Una causa de índole emocional que predispondría a dichos cuadros sería, según la Escuela de Psicosomáticas de París, la «alexitimia» o dificultad del individuo para identificar los sentimientos y para distinguir a éstos de las sensaciones corporales. ${ }^{7,8}$ En nuestro paciente se identificó un fondo alexitímico, el cual fue abordado satisfactoriamente desde la terapia psicoanalítica. Prácticamente un año y medio después, la disnea ha desaparecido y el paciente retomó la práctica del montañismo.

Sr. Editor, los cuadros psicosomáticos se hallan dentro de las alteraciones psiquiátricas más comunes, oscilando su prevalencia en la consulta médica entre un 8 al 15\%.,10 Quienes los padecen suelen hacer uso desproporcionado de los recursos sanitarios, habitualmente se hallan insatisfechos de la atención médica recibida y a menudo tienden a resistirse a la derivación psicológica o psiquiátrica. ${ }^{11}$

En este contexto, consideramos que este caso ejemplifica claramente respecto a causas psicosomáticas de disnea, las cuales deberían ser consideradas y, eventualmente, abordadas en todo paciente clínicamente sintomático sin una base orgánica evidente.

\section{REFERENCIAS}

1. American Thoracic Society. Dyspnea. Mechanisms, assessment and management: a consensus statement. Am J Respir Crit Care Med 1999;159(1):321-340.

2. Neuman A, Gunnbjörnsdottir M, Tunsäter $A$, et al. Dyspnea in relation to symptoms of anxiety and depression: A prospective population study. Respir Med 2006;100(10):1843-1849. 
3. Bass C, Gardner WN. Respiratory and psychiatric abnormalities in chronic symptomatic hyperventilation. Br Med J (Clin Res Ed) 1985;290(6479):1387-1390.

4. Heim E, Blaser A, Waidelich E. Dyspnea: psychophysiologic relationships. Psychosom Med 1972;34(5):405-423.

5. Han JN, Zhu YJ, Li SW, et al. Medically unexplained dyspnea: psychophysiological characteristics and role of breathing therapy. Chin Med J (Engl) 2004;117(1): 6-13.

6. Kellner R. Psychosomatic syndromes, somatization and somatoform disorders. Psychother Psychosom 1994;61(12):4-24.

7. Bach $\mathrm{M}$, Bach D. Alexithymia in somatoform disorder and somatic disease: a comparative study. Psychother Psychosom 1996;65(3):150-152.
8. Lumley MA. Alexithymia and negative emotional conditions. J Psychosom Res 2000;49(1):51-54.

9. Jackson JL, Kroenke K. Prevalence, impact, and prognosis of multisomatoform disorder in primary care: a 5-year follow-up study. Psychosom Med 2008;70(4):430-434.

10. Kroenke K, Rosmalen JG. Symptoms, syndromes, and the value of psychiatric diagnostics in patients who have functional somatic disorders. Med Clin North Am 2006;90(4):603626.

11. Barsky AJ, Orav EJ, Bates DW. Somatization increases medical utilization and costs independent of psychiatric and medical comorbidity. Arch Gen Psychiatry 2005;62(8):903-910.

\section{Atentamente}

Viviana Fiszson-Herzberg, * Pablo

Rubinstein-Aguñin.

*Lic. en Psicología. Equipo de Psicología, Clínica del Vallés (idcsalud). Sabadell, Barcelona, España.

*Médico neumólogo. Servicio de Neumonología, Hospital General de Cataluña (idcsalud). Sant Cugat del Vallés, Barcelona, España.

\section{$\triangle$ Correspondencia:}

Lic. Viviana Fiszson. Equipo de Psicología Clínica del Vallés (idcsalud). Passeig Rubió Ors, 23 (08203) Sabadell, Barcelona. España Teléfono: +34-937283 100-Fax: +34-937 281198 Correo electrónico: vifiszson@yahoo.es 